\title{
Photometric and spectroscopic observations of Sycorax, satellite of Uranus ${ }^{\star}$
}

\author{
J. Romon ${ }^{1}$, C. de Bergh ${ }^{1}$, M. A. Barucci ${ }^{1}$, A. Doressoundiram ${ }^{1}$, \\ J.-G. Cuby ${ }^{2}$, A. Le Bras ${ }^{1,3}$, S. Douté ${ }^{4}$, and B. Schmitt ${ }^{4}$ \\ 1 Observatoire de Paris, Meudon, France \\ 2 ESO, Chile \\ 3 IAS, Orsay, France \\ 4 Lab. de Planétologie de Grenoble, St Martin d'Hères, France
}

Received 9 May 2001 / Accepted 26 June 2001

\begin{abstract}
Sycorax is the brightest of the five irregular Uranian satellites recently discovered. These satellites are supposed to be captured bodies. We present visible and near-infrared photometry, as well as near-infrared spectroscopy of Sycorax. The overall shape of the spectrum is quite puzzling: it has a red slope in the visible (such as Centaurs and Transneptunian objects), whereas the reflectivity strongly decreases beyond $1 \mu \mathrm{m}$ and is rather flat over the near-infrared range. We were not able to reproduce the spectral behaviour of Sycorax using simple materials. A rotational effect is suggested to explain the shape of the spectrum. BVRIJ magnitudes have been measured over a period of 1 hour, with five $V$ measurements which do not show any strong variation. So only a strong change in the magnitude between the $V$ measurements and the $J$ measurement (30 min later) could explain the shape of the spectrum, but further investigation is required to conclude.
\end{abstract}

Key words. planets and satellites: general - planets and satellites: individual: Sycorax

\section{Introduction}

Sycorax (1997 U2) is the brightest of the five irregular satellites of Uranus recently discovered by Gladman et al. $(1998,2000)$. It is a very small object, orbiting far from Uranus on a retrograde orbit (Table 1).

These five satellites are the first irregular satellites detected around Uranus. The so-called irregular satellites of giant planets are satellites located far from the planet on highly inclined elongated orbits, whereas regular satellites orbit closer to the planet on nearly circular orbits, with low inclinations. Retrograde orbits are found only among irregular satellites.

Many studies have been published about the origin of the irregular satellites of Jupiter and Saturn. It is now generally accepted that temporary captures by giant planets can occur. We know several examples of temporarily captured comets, such as the famous Shoemaker-Levy 9. However, it is much more difficult to get stable orbits, as this requires an efficient way of energy dissipation.

Send offprint requests to: J. Romon,

e-mail: jennifer.romon@obspm.fr

* Based on observations made with ESO Telescopes at the Paranal Observatory (under programme IDs 63.S-0200 and 65.S-0147) and with Telescopio Nazionale Galileo at Observatorio del Roque de Los Muchachos.
Table 1. Physical and orbital properties of Sycorax $\left(R_{\mathrm{U}}=\right.$ Uranian radius).

\begin{aligned} & \hline Semi-major axis $481 R_{\mathrm{U}} \\ &$ Eccentricity 0.509 \\ & Inclination $152.7^{\circ} \\ &$ Orbital period 1289 days \\ & \hline\end{aligned}

Pollack et al. (1979) suggested that some satellites may have been captured by gas drag in the protoplanetary nebula. This process could explain the origin of the irregular satellites of Jupiter and Saturn. According to Pollack et al., such a process must have taken place shortly before the end of planetary formation. Another scenario was proposed by Brunini (1995) who suggested that, during the last stages of accretion, the giant planets could have locked temporary captured planetesimals on stable orbits, by changing the shape of their Hill sphere during the temporary capture. This requires a high accretion rate, as well as a rapid migration of the planet, which could both favour this process of permanent capture.

In the case of Uranus, the large obliquity of its spin axis has also to be taken into account. It has been suggested that a collision could have occurred at the end of Uranus's formation that could explain the large obliquity 
(see e.g. Parisi \& Brunini 1997). The existence of irregular satellites orbiting around Uranus could give us constraints about the collision hypothesis (Brunini et al. 2001).

In addition to dynamical studies of these irregular satellites, the knowledge of their physical and chemical properties could give us information about their origin and evolution. $B, R$, and $I$ magnitudes have been obtained by Gladman et al. (1998) for the two largest of these satellites, Sycorax and Caliban. Gladman et al. deduced from these measurements that the two objects were red, and suggested that they could come from the Kuiper Belt, since most Trans-Neptunian Objects (TNOs) are known to be very red. In this paper, we present refined photometric measurements of Sycorax in the visible, as well as some complementary photometric and spectroscopic measurements in the near-infrared.

\section{Observations and data reduction}

\subsection{ISAAC/VLT observations}

Near-infrared photometry and spectroscopy have been performed at the first 8-m Unit (Antu) of the Very Large Telescope (VLT, ESO, Chile), with the ISAAC IR spectroimager in its imaging mode (Morwood et al. 1999; Cuby et al. 2000). In its SW mode (1-2.5 $\mu \mathrm{m}$ spectral range), ISAAC is equipped with a $1024 \times 1024 \mathrm{Hg}$ :Cd:Te array, and has a field of view of $2.5^{\prime} \times 2.5^{\prime}$.

\subsubsection{Near-infrared photometry}

$J, H$, and $K$ s photometry has been performed on 24 th May 1999 . We used the $J, H$, and $K$ s filters, respectively centered at $1.25,1.65$, and $2.16 \mu \mathrm{m}$. Exposure times were $5 \mathrm{~min}$ in $J$ filter, and $6 \mathrm{~min}$ in $H$ and $K$ s filters.

Observations were carried out using the jitter imaging technique, as usual in the infrared, that is, applying telescope offsets between frames (dither pattern). Data reduction was carried out with both the ESO-MIDAS and eclipse (Devillard 1997) data reduction packages. A combined image was generated using the jitter routine from the eclipse package. The main steps of this procedure are: i) flatfielding, using twilight flats, ii) estimate of a sky frame by median filtering of the individual frames in the stack, after scaling with the median of the number of counts in each image to account for sky intensity variations, iii) subtraction of the sky frame from all the individual frames, after proper scaling, iv) determination of the offsets between frames, based on approximate values present in the FITS header and using an auto-correlation technique, v) final image registration. More details about the routine can be found at http://www.eso.org/eclipse/.

The magnitude of Sycorax was then measured using classical aperture photometry for each filter. The results are presented in Table 2 . The zero points of the photometric calibration were measured from observations of S889-E, from the HST/Nicmos list of faint IR standard stars.
Table 2. Magnitudes of Sycorax in visible and near-infrared filters.

\begin{tabular}{llll}
\hline Date - Time (UT) & Instrument & Filter & Magnitude \\
\hline 24th May 1999 05:17 & ISAAC & $J$ & $19.53 \pm 0.10$ \\
22th Aug. 2000 01:19 & ISAAC & $J_{\mathrm{S}}$ & $19.72 \pm 0.10$ \\
9th Aug. 2000 23:58 & ISAAC & $J_{\mathrm{S}}$ & $19.74 \pm 0.15$ \\
24th May 1999 08:35 & ISAAC & $H$ & $19.28 \pm 0.05$ \\
24th May 1999 08:43 & ISAAC & $K \mathrm{~s}$ & $19.07 \pm 0.05$ \\
\hline 8th Aug. 2000 01:45 & OIG & $B$ & $21.48 \pm 0.07$ \\
8th Aug. 2000 01:36 & OIG & $V$ & $20.74 \pm 0.06$ \\
8th Aug. 2000 01:40 & OIG & $V$ & $20.76 \pm 0.07$ \\
8th Aug. 2000 01:49 & OIG & $V$ & $20.77 \pm 0.07$ \\
8th Aug. 2000 01:54 & OIG & $V$ & $20.74 \pm 0.06$ \\
8th Aug. 2000 02:01 & OIG & $V$ & $20.72 \pm 0.06$ \\
8th Aug. 2000 01:52 & OIG & $R$ & $20.24 \pm 0.03$ \\
8th Aug. 2000 01:57 & OIG & $I$ & $19.68 \pm 0.02$ \\
\hline 8th Aug. 2000 02:33 & ARNICA & $J$ & $19.63 \pm 0.15$ \\
\hline
\end{tabular}

Table 3. Characteristics of the $J$ filters used (for comparison of the different measurements of the $J$ magnitude).

\begin{tabular}{cccc}
\hline Instrument & Filter & Central wav. $(\mu \mathrm{m})$ & Bandwidth $(\mu \mathrm{m})$ \\
\hline ISAAC & $J$ & 1.25 & 0.29 \\
ISAAC & $J \mathrm{~s}$ & 1.24 & 0.16 \\
ARNICA & $J$ & 1.251 & 0.27 \\
\hline
\end{tabular}

Table 4. Description of the spectroscopic observations.

\begin{tabular}{ccccc}
\hline Date (UT) & $\begin{array}{c}\text { Spectral } \\
\text { range }\end{array}$ & Exp. Time & Airmass & Calib. \\
& & & \\
\hline May 24 1999 & $1.1-1.4$ & $1 \times 48 \mathrm{~min}$ & $1.01-1.04$ & 128 \\
08:56:21 & $\mu \mathrm{m}$ & & & Nemesis \\
\hline Aug. 22 2000 & $1.45-1.8$ & $4 \times 48 \mathrm{~min}$ & $1.01-1.17$ & $\mathrm{HD}$ \\
01:51:09 & $\mu \mathrm{m}$ & & & 1835 \\
\hline Sep. 07 2000 & $1.9-2.45$ & $6 \times 48 \mathrm{~min}$ & $1.01-1.53$ & $\mathrm{HD}$ \\
$00: 28: 00$ & $\mu \mathrm{m}$ & & & 144585 \\
\hline
\end{tabular}

Further photometry was achieved in the $J_{\mathrm{S}}$ filter by using spectroscopic acquisition images. The characteristics of the ISAAC $J$ and $J_{\mathrm{S}}$ and ARNICA filters are given in Table 3 .

\subsubsection{Near-infrared spectroscopy}

The spectroscopic observations took place at the VLTAntu, in May 1999 and August-September 2000. The slit width was 1 arcsec, providing a spectral resolution $R$ of $\sim 500$. Three spectral domains corresponding to the $J, H$, and $K$ bands were covered separately (see Table 4 ). The object was nodded along the slit, between 2 positions $A$ and $B$. In addition, some jittering was added around each of the $A$ and $B$ positions (except in May 1999), to allow a better removal of bad pixels. Between 4 and 6 images were 
taken at each $A$ or $B$ position (one cycle), and between 1 and $6 A B$ or $B A$ cycles were executed sequentially, depending on the spectral range and integration time (see Table 4).

The data reduction was carried out, as for the imaging, with the ESO-MIDAS and eclipse packages. The frames were first flatfielded and corrected for distortions (spatial and spectral). The frames in the $J$ band obtained in May 1999 were further corrected for bad pixels. Then $A$ and $B$ images belonging to one $A B$ or $B A$ cycle are averaged and subtracted. All $A-B$ images from one sequence are then registered taking into account the telescope offsets as recorded in the FITS headers, and combined. The resultant image has one positive and one negative spectrum of the object, and some sky residuals resulting from the sky variations. The $A-B$ image is then combined, after adequate offsetting, with the $B-A$ image: this superimposes all the useful signal corresponding to the $A$ and $B$ positions, while removing the sky residuals. Some offsets (in wavelength) were occasionally noted within a sequence of frames, which were corrected for. All final images corresponding to different sequences with the same filter were then combined together, from which the object spectrum was finally extracted and wavelength calibrated using Xe and $\mathrm{Ar}$ arc frames taken in the morning following the observations.

Further processing was done for the removal of the telluric and solar features, dividing the Sycorax spectra by spectra of solar analogs: HD 1835 (G3V) and HD 144585 (G5V) (Hardorp 1978). A C-type asteroid (128 Nemesis) was also observed and its spectrum was used for removal of the telluric and solar features. C-types asteroids are known to have featureless and generally flat spectra in the nearinfrared range, so they can be considered as good solar analogs.

The resulting spectra were finally smoothed with a Gaussian filter using $\sigma=30$ pixels, degrading the final spectral resolution to $\sim 50$ (see Fig. 1). The final signalto-noise ratio obtained is about 20 to 30 , depending on wavelength.

\subsection{TNG observations - BVRI and J photometry}

$B V R I$ and $J$ photometry of Sycorax has been carried out on 8th August 2000 at the Observatorio del Roque de los Muchachos (La Palma, Canary Islands). Observations were performed using the Telescopio Nazionale Galileo (TNG). TNG is a $3.5-\mathrm{m}$ alt-azimuth telescope, with two Nasmyth foci and active optics control.

For the visible photometry, we used the Optical Imager Galileo (OIG) CCD camera mounted on the Nasmyth A focus. For the infrared photometry, we used the ARNICA near-infrared camera mounted on the same focus. This configuration allowed us to switch from OIG to ARNICA in order to obtain quasi-simultaneous visible and nearinfrared observations, and avoid rotational effects. Details about the observations are given in Table 2 .

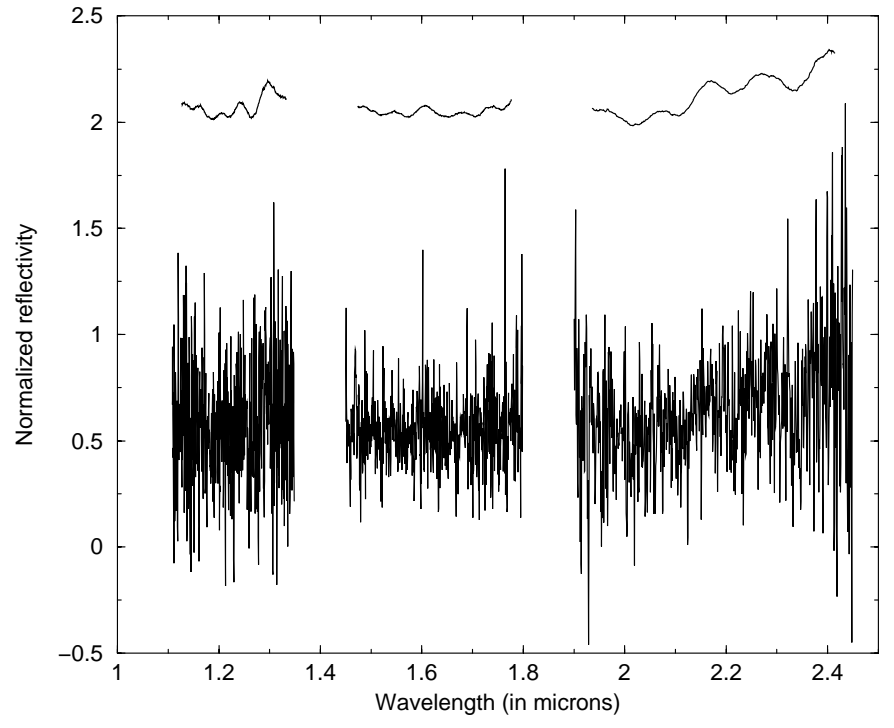

Fig. 1. Spectral reflectivity of Sycorax in the $J, H$, and $K$ ranges. The spectra have been adjusted using the colors derived from the photometric measurements. The lower curves correspond to the full resolution spectra $(R=500)$, whereas the upper curves show the same spectra at a spectral resolution of 50 . The upper curves have been offset by 1.5 .

\subsubsection{Visible photometry}

The OIG CCD camera is equiped with two $2048 \times 4096$ pixels chips, and has a field of view of $4.9^{\prime} \times 4.9^{\prime}$ (binning $2 \times 2$ ). We used Bessel $B, V$, Cousins $R$, and Moult $I$ filters, centered respectively at $436 \mathrm{~nm}, 533 \mathrm{~nm}, 625 \mathrm{~nm}$, and $825 \mathrm{~nm}$. Exposure times were $60 \mathrm{~s}$ for $V$ and $R$ filters, and $120 \mathrm{~s}$ for $B$ and $I$ filters.

Data reduction was performed using MIDAS software. Frames were first corrected from bias and flat-field. Then, instrumental magnitudes were measured using specific data reduction techniques developed for observations of TNOs (see Barucci et al. 2000 for details about the data reduction techniques used). Photometric calibration was performed using the usual calibration technique: 7 standard stars were observed at different airmasses during the night; zero-point and extinction coefficients were computed using a least square method. The resulting magnitudes are reported in Table 2 . We compared the measurements previously published with our data, which are more accurate. Gladman et al. (1998) obtained for Sycorax visible colors of $B-R=1.6 \pm 0.2$ and $R-I=0.6 \pm 0.2$. Our measurement of the $R-I$ color index $(R-I=0.57 \pm 0.04)$ is fully consistent with the previous one, whereas our $B-R$ value is lower $(B-R=1.23 \pm 0.08)$.

\subsection{2. $J$ photometry}

The ARNICA near-infrared camera is a $\mathrm{HgTeCd}$ array detector $\left(256 \times 256\right.$, i.e. a field of view of $\left.90^{\prime \prime} \times 90^{\prime \prime}\right)$. We used the $J$ broadband filter, centred at $1.251 \mu \mathrm{m}$ (see Table 3 ). Exposure time was $4 \mathrm{~min}$ (four frames of $1 \mathrm{~min}$, each frame being the co-average of four $15 \mathrm{~s}$-frames). Observations 


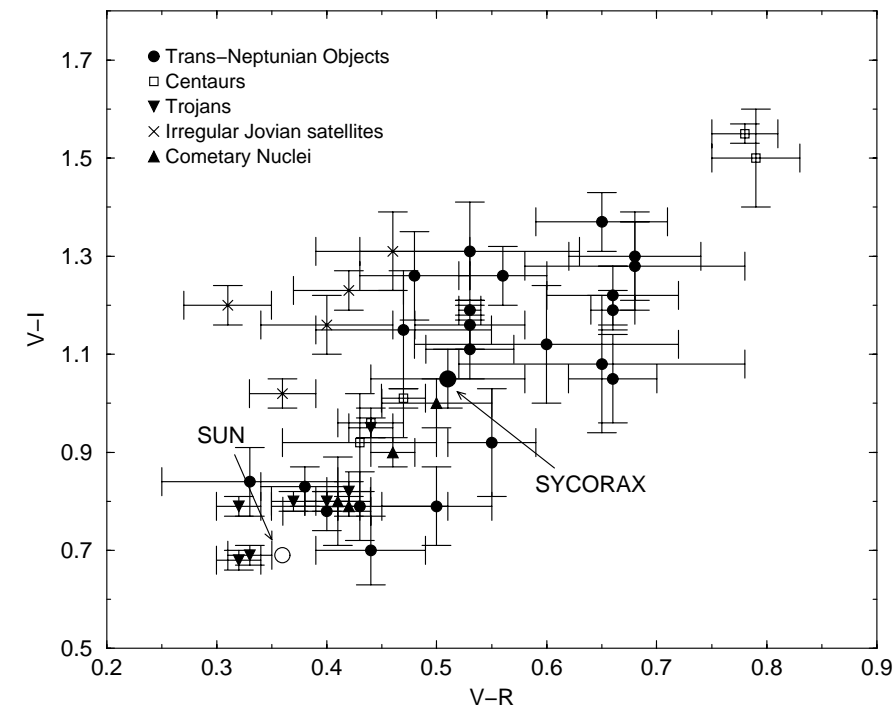

Fig. 2. $V-R$ vs. $V-I$ color plot. This is a comparison of different types of small bodies in the Solar System: TransNeptunian Objects (Barucci et al. 1999; Barucci et al. 2000; Doressoundiram et al. 2001), Cometary nuclei (Thomas \& Keller 1989; Luu 1993; Lamy et al. 2001), Trojans (Degewij \& Van Houten 1979), Centaurs (Davies 2001), Irregular jovian satellites (Luu 1991), Sycorax (this work). The solar colors are shown for comparison (Hardorp 1980; Hartmann et al. 1982).

were carried out using the jitter imaging technique. Data reduction was performed using both IRAF and MIDAS. The steps of the data reduction process are roughly the same as those used for VLT near-infrared photometric observations (see 2.1.1). Instrumental magnitudes were then obtained from the resulting frames using the MIDAS procedures developped for the visible observations (see 2.2.1). Calibration of the instrumental response was obtained by monitoring standard star fields at different airmasses (Hunt et al. 1998).

\section{Results and discussion}

These new photometric results complete $B R I$ data previously obtained by Gladman et al. (1998). Sycorax spectrum has a strong red slope in the visible range: this is roughly the same behaviour as TNOs and Centaurs. Figure 2 shows a color plot $(V-R$ vs. $V-I)$ of Sycorax compared to other small bodies (TNOs, Centaurs, cometary nuclei, Trojans, and irregular Jovian satellites). Sycorax colors are more similar to those of TNOs, Centaurs, and cometary nuclei, than to those of Trojans, and irregular Jovian satellites. Such a common behaviour among Centaurs, comets, and TNOs is not surprising as Centaurs and short-period comets are believed to come from the Kuiper Belt. Sycorax colors seem to be intermediate between those of neutral and very red TNOs and Centaurs. The visible and near-infrared colors of Sycorax are given in Table 5 .

We have also compared the visible colors of Sycorax with those of regular satellites of Uranus:

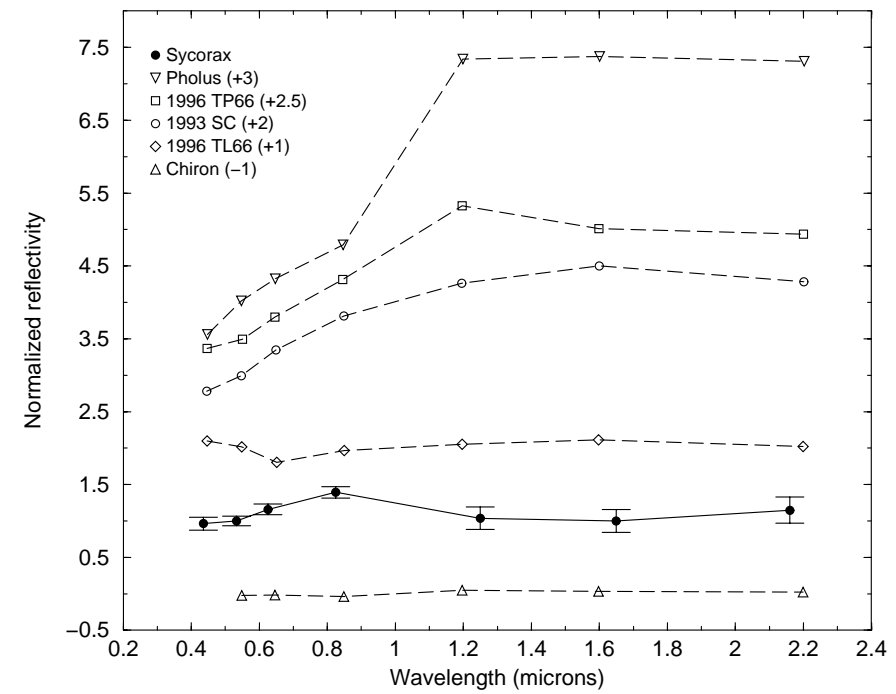

Fig. 3. Reflectivity of Sycorax, compared to those of some TNOs and Centaurs (from Jewitt \& Luu 1998). Solar contribution has been removed using the solar colors (Hardorp 1980; Hartmann et al. 1982). The reflectivity has been computed using the different estimates of the $J$ magnitude, in order to minimize the rotational effect on the reflectivity curve. Sycorax spectrum is very different from those of TNOs and Centaurs, especially around $0.8-1.2 \mu \mathrm{m}$. The reflectivity has been normalized to 1 at $0.55 \mu \mathrm{m}$.

Karkoschka (1997) found that the major satellites of Uranus have visible colors that range from slightly bluer than the F-type asteroids (negative gradient) to slightly redder than the D-type asteroids ${ }^{1}$. So the major regular satellites of Uranus appear to be bluer than Sycorax. JHK measurements of some regular satellites have recently been obtained by Trilling \& Brown (2000): several appear to have flat spectra in the near-infrared range, as seems to be the case for Sycorax and many other objects among TNOs and Centaurs, in this spectral range.

Sycorax spectral behaviour from the visible to the near-infrared ranges is much more unusual. The reflectivity increases in the visible range, whereas it strongly decreases from 0.8 to $1.2 \mu \mathrm{m}$, and seems to be flat from 1.2 to $2 \mu \mathrm{m}$. Such a spectral behaviour is very different from that of TNOs and Centaurs: none of the spectra already obtained show such a strong difference between the visible and near-infrared range (see Fig. 3).

Since the whole data set has not been obtained simultaneously, a possible rotational effect could affect the reflectivity curve. However, we first assumed that no strong rotational effect affects the reflectivity curve, and we tried to investigate the materials which may contribute to the spectrum of Sycorax. We ran a radiative transfer model (Douté \& Schmitt 1998), considering simple geographical mixtures of organics (kerogen, Titan tholin, amorphous carbon), minerals (pyroxene and olivine), and water ice (Grundy \& Schmitt 1998). Titan tholins (Khare et al. 1984) are complex organic solids that have already been

\footnotetext{
1 The D-type asteroids include mainly the Trojans.
} 

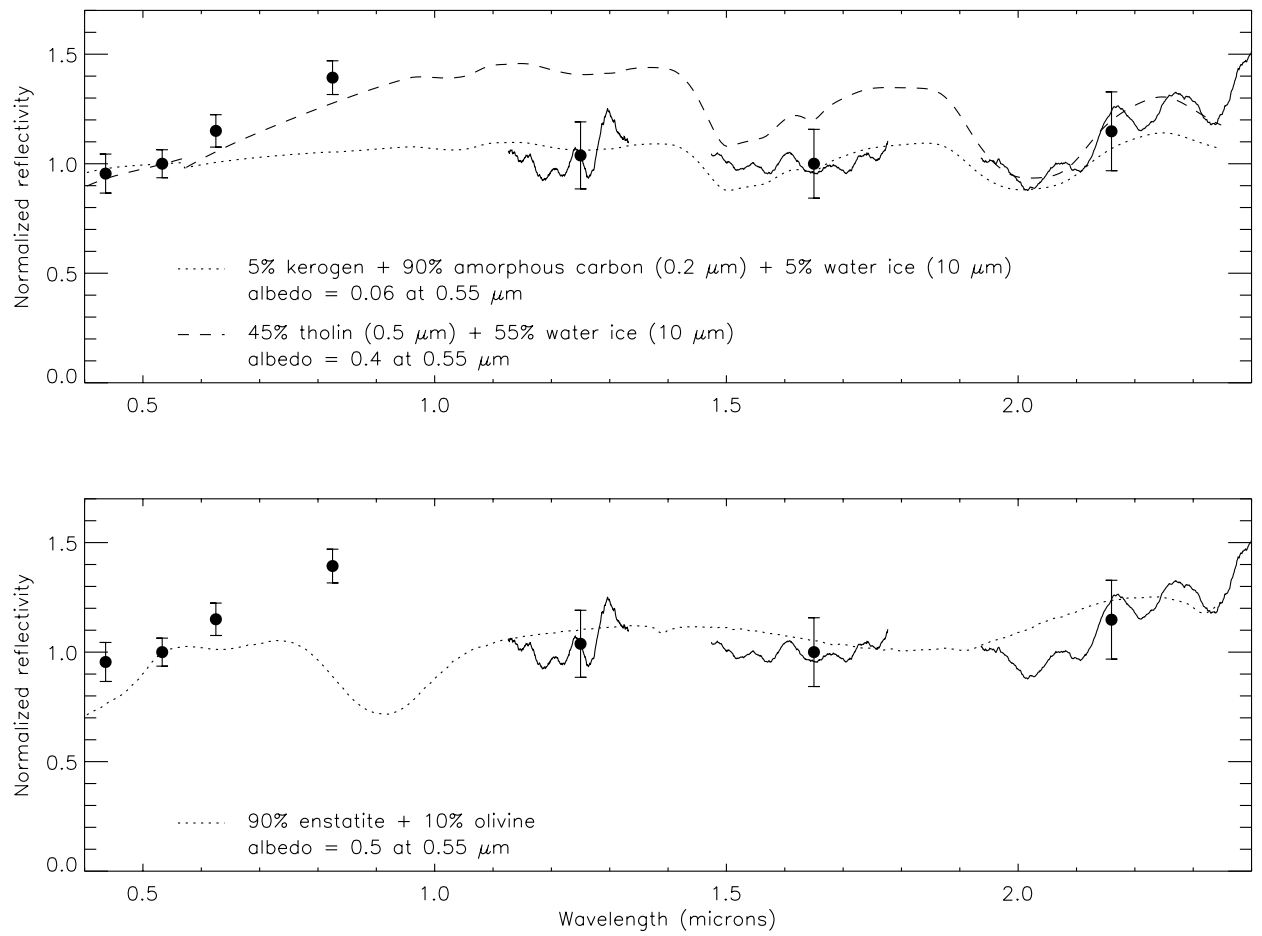

Fig. 4. Some attempts in modelling the spectrum of Sycorax. None of these models have been fully satisfactory in reproducing the overall shape of the spectrum. Especially, we could not reproduce both the high reflectivity level at $0.8 \mu \mathrm{m}$ and the low level at $1.25 \mu \mathrm{m}$. The corresponding albedo has been indicated for each model.

Table 5. Colors of Sycorax. The $J-H$ and the $J-K$ s colors have been computed using the VLT photometry (May 1999), whereas the other ones have been computed using the TNG photometry (Aug. 2000). The average of the $V$ measurements, as well as the absolute $H_{V}$ magnitude are also given. Solar colors: $B-V=0.76, V-R=0.36, V-I=0.69, V-J=1.08$, $J-H=0.29, J-K \mathrm{~s}=0.35$ (Hardorp 1980; Hartmann et al. 1982).

\begin{tabular}{lll|l}
\hline$B-V$ & $=$ & $0.71 \pm 0.10$ & \\
$V-R$ & $=$ & $0.52 \pm 0.07$ & $\bar{V}=20.75 \pm 0.06$ \\
$V-I$ & $=$ & $1.05 \pm 0.06$ & \\
$V-J$ & $=1.12 \pm 0.16$ & $H_{V}=7.83 \pm 0.06$ \\
$J-H$ & $=0.25 \pm 0.11$ & \\
$J-K \mathrm{~s}$ & $=0.46 \pm 0.16$ & \\
\hline
\end{tabular}

used to explain the red slope observed on the spectrum of Centaur 5145 Pholus, from 0.4 to $1 \mu \mathrm{m}$ (see Cruikshank et al. 1998). Kerogen-like compounds (Clark et al. 1993) constitute most of the organic matter of carbonaceous meteorites (these compounds are also called macromolecular carbon). They have been suggested by Gradie \& Veverka (1980) to explain spectra of D-type asteroids. Amorphous carbon (Zubko et al. 1996) is a dark featureless compound often included in models of $\mathrm{dark}^{2}$ solar system objects. Figure 4 shows different models that we have investigated. The upper figure shows two models that could both fit

\footnotetext{
2 Since we do not presently have any constraint on Sycorax albedo, we cannot exclude that Sycorax is a very dark object (as most of the small bodies in the outer solar system) as well as a bright one (as some satellites of the outer planets).
}

the overall behaviour around $2 \mu \mathrm{m}$. But we could not find any mixture of simple organic compounds and water ice that could fit both the spectrum around $0.8 \mu \mathrm{m}$ and that around $1.25 \mu \mathrm{m}$. We have also investigated mixtures including silicates to check if they could better fit the spectrum. The mixture pyroxene + olivine $^{3}$ could fit the spectrum beyond $1.2 \mu \mathrm{m}$, but does not fit at all the visible part of the spectrum.

The first attempt in modelling Sycorax spectrum did not therefore allow us to find a mixture that could fit the overall shape of the spectrum. To fit both the high reflectivity at $0.8 \mu \mathrm{m}$ and the low value at $1.25 \mu \mathrm{m}$ is not obvious using simple mixtures.

The whole data set presented here was not obtained simultaneously (see Tables 2 and 4), so these observations could correspond to different areas on Sycorax surface, and we have to consider a possible rotational effect that could affect the reflectivity curve. In particular, the JHKs photometry (VLT, May 1999) was not performed simultaneously ( 3 hours between the $J$ and the $H K$ s measurements).

The TNG BVRIJ photometry was obtained over a period of 1 hour: a $V-B-V-R-V-I-V$ sequence was carried out during $25 \mathrm{~min}$, and the $J$ measurement was performed 32 min later. Small variations of the $V$ magnitude appear in the measurements, but they are not significant since they are within the error bars. A strong

${ }^{3}$ The spectra of pyroxene (Ref. 128288) and olivine (Ref. 137044) are from the USGS spectral library (Clark et al. 1993). 
variation of the magnitude within the next $32 \mathrm{~min}$ would introduce an error in the computed $V-J$ color. If the $V$ magnitude strongly increases within the $32 \mathrm{~min}$, the $V-J$ color index would increase as well, and the relative reflectivity in the $J$ band may increase enough to equal that in the $I$ band. So the model tholin + water ice (see Fig. 4) could fit the spectrum. Such a strong variation in the magnitude could be explained by different effects: a strong change in the albedo over the surface, an elongated shape for the satellite, ... But for the moment, we are not able to say if such a change really occurred.

\section{Conclusion}

This work is the first exploration of the physical properties of the irregular Uranian satellites. Sycorax appears to be a very interesting object: the overall shape of its spectrum is very curious, and does not look like what we expected to find since its visible colors were very close to those of TNOs and Centaurs. Does what we found really correspond to the composition of Sycorax surface? Or is it due to some kind of rotational effect? From our data, a strong change in the magnitude may explain the observed shape of the spectrum. But the rotational period is still unknown, so we are unable to say if it occurred. A more complete investigation of Sycorax is absolutely required to better understand this satellite.

For the moment, Sycorax is the only one of the irregular Uranian satellites that can be fully studied with photometry and spectroscopy techniques. The four other satellites are fainter than 23th magnitude, so spectroscopic observations cannot be carried out, even with the largest telescopes available. But the knowledge of their colors and rotational periods could help us in constraining the possible links between these five satellites.

Acknowledgements. We would like to thank E. Le Floc'h for fruitful discussions about near-infrared data reduction.

\section{References}

Barucci, M. A., Doressoundiram, A., Tholen, D. J., et al. 1999, Icarus, 142,476
Barucci, M. A., Romon, J., Doressoundiram, A., et al. 2000, AJ, 120,496

Brunini, A. 1995, Earth, Moon and Planets, 71, 281

Brunini, A., Parisi, M. G., \& Tancredi, G. 2001, Icarus, submitted

Clark, R. N., Swayze, G. A., Gallagher, A. J., et al. 1993, U.S. Geological Survey, Open File Report 93-592

Cruikshank, D. P., Roush, T. L., \& Bartholomew, M. J. 1998, Icarus, 135, 389

Cuby, J.-G., et al. 2000, The Messenger, 101

Davies, J. K. 2001, Physical Characteristics of TransNeptunian Objects and Centaurs, in Minor Bodies in the Outer Solar System, ed. A. Fitzsimmons, D. Jewitt, \& R. M. West, ESO Astrophysics Symposia (Springer), 9

Degewij, D., \& Van Houten, C. J. 1979, Distant asteroids and outer jovian satellites, in Asteroids, ed. T. Gehrels (Univ. of Arizona Press), 417

Devillard, N. 1997, The Messenger, 87

Doressoundiram, A., Barucci, M. A., Romon, J., et al. 2001, Icarus, submitted

Douté, S., \& Schmitt, B. 1998, J. Geophys. Res. E, 103(25), 809

Gladman, B. J., Nicholson, P. D., Burns, J. A., et al. 1998, Nature, 392, 897

Gladman, B., Kavelaars, J. J., Holman, M., et al. 2000, Icarus, 147,320

Gradie, J., \& Veverka, J. 1980, Nature, 283, 840

Grundy, W., \& Schmitt, B. 1998, JGR E, 103, 31367

Hardorp, J. 1978, A\&A, 63, 383

Hardorp, J. 1980, A\&A, 91, 221

Hartmann, W. K., Cruikshank, D. P., \& Degewij, J. 1982, Icarus, 52, 377

Hunt, L. K., Mannucci, F., Testi, L., et al. 1998, AJ, 115, 2594

Jewitt, D. C., \& Luu, J. X. 1998, AJ, 115, 1667

Karkoschka, E. 1997, Icarus, 125, 348

Khare, B., Sagan, C., Arakawa, E. T., et al. 1984, Icarus, 60, 13

Lamy, P. L., et al. 2001, Icarus, Submitted

Luu, J. X. 1991, AJ, 102, 1213

Luu, J. X. 1993, Icarus, 104, 138

Morwood, A., et al. 1999, The Messenger, 95, 1

Parisi, M. G., \& Brunini, A. 1997, Planet. Sp. Sci., 45, 181

Pollack, J. B., Burns, J. A., \& Tauber, M. E. 1979, Icarus, 37,587

Thomas, N., \& Keller, H. U. 1989, A\&A, 213, 487

Trilling, D. E., \& Brown, R. H. 2000, Icarus, 148, 301

Zubko, V. G., Mennella, V., Colangeli, L., et al. 1996, MNRAS, 282, 1321 